\title{
Forward-Light-Scattering Characterization of Pre-Crystalline Aggregates in Crystallizing Lysozyme Solutions
}

\author{
Takashi Wakamatsu \\ Department of Electrical and Electronic System Engineering, Ibaraki National College of Technology, Ibaraki, \\ Japan \\ Email: wakamatu@ee.ibaraki-ct.ac.jp
}

Received 25 April 2014; revised 28 May 2014; accepted 15 June 2014

Copyright (C) 2014 by author and Scientific Research Publishing Inc.

This work is licensed under the Creative Commons Attribution International License (CC BY). http://creativecommons.org/licenses/by/4.0/

(c) (i) Open Access

\begin{abstract}
We present a method to characterize lysozyme pre-crystalline aggregates using a forward-lightscattering technique, which is highly sensitive to protein aggregates. The static light scattering properties at small angles of crystallizing lysozyme aggregates are discussed, and related to the crystallization conditions based on the concentration of added precipitant $\mathrm{NaCl}$. Lysozyme solutions that started to crystallize because of precipitant exhibited profiles of forward light scattering that could be fitted by non-integer power laws, which indicated fractal aggregations of lysozyme had formed. Pre-crystalline solutions, in which lysozyme crystals later grew, had dense structural fractal clusters with fractal dimensions of $D>1$.5. In contrast, solutions with aggregates in which crystals did not grow, had forward-light-scattering profiles that deviated from a power law or had lower power values.
\end{abstract}

\section{Keywords}

Protein Crystallization, Lysozyme, Light Scattering, Fractal Aggregation, Power Law

\section{Introduction}

Structure analysis of proteins by x-ray or neutron diffraction provides a fundamental and useful database for developing new functional materials such as innovative drugs and high quality processed foods. In protein crystallography, crystallization of the protein is the main bottleneck because it relies on trial and error procedures [1] [2]. In solutions of lysozyme as a model protein, it is well accepted that liquid-liquid phase separation and solid-liquid phase separation coexist in a metastable region of phase diagrams [3]-[5]. Protein-rich droplets formed 
from the liquid-liquid phase separation have been observed, and lysozyme crystals have grown in these solutions [4]. However, an occurrence mechanism of the metastable liquid-liquid phase separation for protein crystallization is not well understood in detail. There are few methods for evaluating crystallizing protein solutions, and as such, details of the protein crystallization process remain unknown.

Formation of protein clusters $100 \mathrm{~nm}-1 \mu \mathrm{m}$ in size that remain in solution is an important pre-crystalline process [6] [7]. Light scattering techniques, particularly dynamic light scattering (DLS), has frequently been used to characterize protein aggregates [7]-[12]. DLS and static light scattering (SLS) studies demonstrated by Georgalis et al. have revealed that lysozymes form fractal cluster aggregates before crystallizing [13] [14]. Similar aggregations of lysozymes have been studied by Tanaka et al., using DLS techniques [7] [15]. However, the relationship between the protein crystallization and the fractal aggregation has been overlooked. Establishing a protein crystallization screening method requires distinguishing formations of aggregates (clusters) that have the potential to grow into crystal nuclei from those that do not.

SLS patterns for fractal aggregates exhibit a power law of the scattering wave vector $q$ : where the scattering intensity, $I_{s}(q) \propto q^{-\alpha}(\alpha>0)$ [16]-[19]. The $q$ is given by $2 k_{0} n \sin (\theta / 2)$, where $n$ is the refractive index of the sample solvent, and $\theta$ is the original scattering angle of the sample solution. The power value $\alpha$ from the equation is equal to the fractal dimension $D$ of the scattering aggregates, which gives an interior parameter of the fractal aggregates [16]. This means that a structure factor $S(q)$ of the aggregates can also be expressed as the power law [16] [17] of $S(q) \propto q^{-D}$. Observation can be made of the power-law SLS patterns in the range of $1 / R_{G} \ll q \ll 1 / a$, where $R_{\mathrm{G}}$ is the average gyration radius of aggregates and $a$ is the size of the minimum scattering unit, such as a monomer or an oligomer [16] [17]. From the power-law formula $I_{s}(q) \propto q^{-\alpha}$, small $q$ values offer larger scattering intensities in the SLS measurements, such that SLS sensitivity to protein aggregation increases at small scattering angles.

Previous work [20] [21] has shown that forward static light scattering (F-SLS) at small angles (typically $\theta_{\mathrm{s}}<$ $8^{\circ}$ ) is highly sensitive to protein aggregates in crystallizing solutions. Small-angle F-LS measurements can characterize protein aggregation directly. In this work, we evaluate lysozyme aggregation in pre-crystalline solutions using a fitting analysis of F-SLS profiles, and discuss the relationship between the fractal aggregation and the crystallization conditions based on the concentration of an added precipitant, $\mathrm{NaCl}$.

\section{Experimental}

\subsection{Sample Preparations and Sample Cells for Measurements}

Hen egg-white lysozyme (HEWL; Seikagaku, six times recrystallized) was used also for this study without further purification [20] [21]. Solutions of HEWL [90 mg/ml (6.3 mM)] and $\mathrm{NaCl}[15 \%(\mathrm{w} / \mathrm{v})(2.56 \mathrm{M})]$ in a sodium acetate buffer ( $50 \mathrm{mM}, \mathrm{pH}=4.6 \pm 0.1)$, and the buffer were prepared separately as stock solutions. The solutions were passed through a filter with $0.2-\mu \mathrm{m}$ pore. Just before FLS measurements, these solutions were mixed at the appropriate rate in a micro test tube $(1.5 \mathrm{ml})$ at $20 \pm 0.5^{\circ} \mathrm{C}$. A sample $(\sim 18 \mu \mathrm{l})$ for the FLS measurements was taken immediately from the test tube and injected into the sample cell.

The sample cell was composed of two microscope cover glass plates (BK-7, $18 \mathrm{~mm} \times 32 \mathrm{~mm}$, and $0.15 \mathrm{~mm}$ thick) and a silicon rubber sheet ( 1.0 mm thick). These components were cleaned with a supersonic wave syringe using a neutral detergent and ethyl alcohol, respectively. The rubber sheet had a circular hole with a diameter of about $6 \mathrm{~mm}$, and was sandwiched between the two cover glass plates. The mixed lysozyme solution was dropped into the circular space between the glass plates. This parallel plate cell allows for precise measurements of the forward light scattering at small angles from the sample solutions.

\subsection{Forward Static Light Scattering (F-SLS) Measurements}

Figure 1 shows a schematic of the setup for F-SLS measurements and photographs of a scattered light around a transmitted light beam from crystallizing lysozyme solutions, which were projected onto a screen far from the solution cell. The slight light in the photograph (a), which extends around the white bright spot of the transmitted light in the center, is the forward scattered light at small angles. This forward light scattering is characteristic of a solution with pre-crystalline lysozyme aggregates and clusters. When precipitates or microcrystals of lysozyme had formed, ring-like diffractions are observed on the forward screen, as shown in the photograph (b), which are distinct from the small amounts of light due to small clusters. 


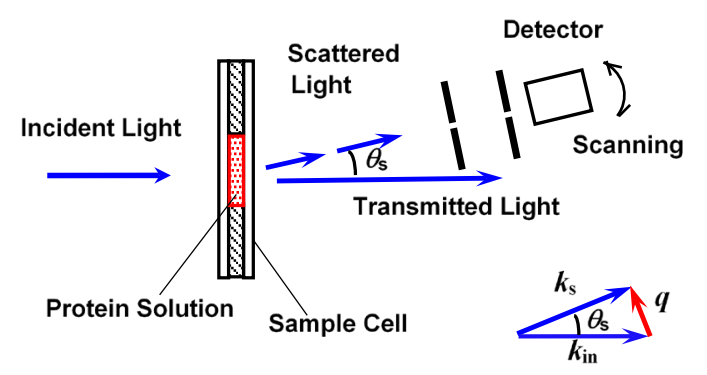

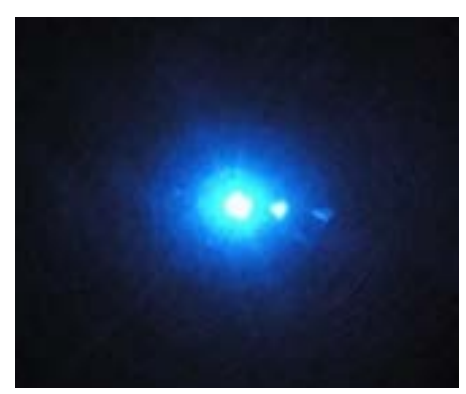

(a)

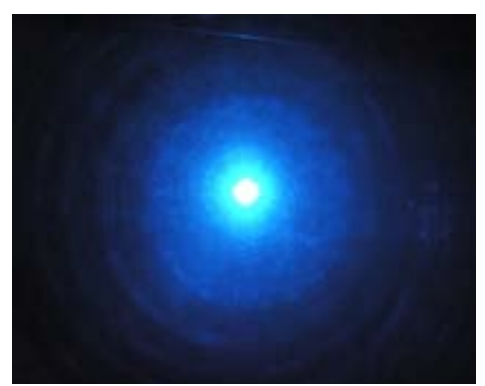

(b)

Figure 1. Schematic of the experimental setup for measuring the forward light scattering (F-LS) of crystallizing protein solutions. Photographs of the forward light scattering projected onto a screen far from the sample solution cell.

The apparatus for F-SLS measurements was constructed in house [20] [21]. The sample cell was mounted on the $\theta$ rotation stage of a $\theta-2 \theta$ coupling stage with a common axis, which was controlled with a personal computer through a controller. A narrow laser beam $\left(\sim 0.7 \mathrm{~mm}\right.$ in diameter, spread angle $\left.<1.2 \times 10^{-3} \mathrm{rad}\right)$ from a diode-pumped solid state laser ( $\lambda=473 \mathrm{~nm}, 25 \mathrm{~mW}$ ) was directed onto the sample cell perpendicularly (at a fixed incident angle of $\theta=0$ ). The incident light was split with a half-mirror and the intensity was monitored using a photo-diode detector, as a reference light for estimation of the relative light scattering intensity. Light scattered from the sample solution was detected using a small solid photomultiplier detector mounted on the $2 \theta$ rotation stage. To measure the scattered light precisely at the scattering angle $\theta_{\mathrm{s}}$, a pair of slits ( $2 \mathrm{~mm}$ in diameter) was positioned on the $2 \theta$ rotation stage at an appropriate distance in front of the detector. The detected scattered light at each angle was recorded on the computer through an analog-digital interface converter.

Light scattering of the buffer solutions was extremely weak and a constant for scattering angles except for in very small angular range $\left(<2^{\circ}\right)$, so that the background scattering of the sample solutions was negligible. Furthermore, scattering pattern of lysozyme small aggregates $<0.2 \mu \mathrm{m}$, which were passed through the $0.2-\mu \mathrm{m}$ filter in the solution preparation, was similar to that of the buffer solutions and the scattering at the small angles can be involved in the background scattering. Thus we estimated the scattering light intensity $I_{\mathrm{s}}$ as the ratio of monitored incident light intensity, and obtained the SLS patterns for the sample solutions from measurements of scattering light intensities by scanning the $2 \theta$ rotation stage at a step angle of $0.1^{\circ}$. The measured $I_{s}\left(\theta_{s}\right)$ was corrected for the measurement angle by multiplying by a factor of $1 / \cos \theta_{s}$, to obtain the accurate scattering angle distribution. In the light scattering measurements for lysozyme solutions before crystallization, the influence of multiple light scatterings can be ignored, because the transmitted light in the sample solutions is strong because of the lack of an absorption at the energy of the probe light $(\lambda=473 \mathrm{~nm})$, while the scattering light intensity is weak, as shown in the photograph (a) of Figure 1. We obtained the scattering distribution in $q$ space, $I_{\mathrm{s}}(q)$ from the SLS measurements. It is worth noting that the scattering measurements allow the estimation of $q=2 k_{0} \sin \left(\theta_{s} / 2\right)$, without considering the index of solvent, because the scattering light from the sample solution is refracted in the cell structure of solution (scatters)/glass plate/air and is measured on the air side through the plane glass cell. The scattering angle range of $2^{\circ}<\theta_{\mathrm{s}}<15^{\circ}$ corresponds to the scattering wave vector of 0.46 $\mu \mathrm{m}^{-1}<q<3.47 \mu \mathrm{m}^{-1}$.

Measuring the forward light scattering, as displayed in the photographs, requires time of the order of minutes when using this experimental setup (Figure 1). For example, to measure the SLS in the scattering angles from $15^{\circ}$ up to $2^{\circ}$ at a scanning step of $0.1^{\circ}$, required about 6 minutes. The addition of the electrolyte $\mathrm{NaCl}$ to the 
HEWL solutions induces aggregation because the electrolyte ions decrease the electrostatic potential screening (Debye-Hückel screening [16]), which results in weakening the repulsive forces between colloidal proteins. Since initial aggregation of lysozyme just after the addition of precipitant is quite drastic [13] [14], the SLS for the HEWL solutions was measured about 30 min after mixing when they had reached a relatively stable state.

\section{Results and Discussion}

\subsection{Crystallization Conditions of Lysozyme}

The crystallization conditions for lysozyme are summarized in Table 1. Two days after the HEWL solutions had been mixed with the precipitant $\mathrm{NaCl}$, we judged whether lysozyme crystals had grown using a microscope $(\times 40)$. Table 1 corresponds to a phase diagram that describes the relationship between concentrations of crystallizing protein and precipitant at a constant temperature and at a constant $\mathrm{pH}$. The results in Table 1 indicate a dissolving area with no crystal growth of protein, a metastable area with crystal growth, and the boundary region. The boundary in lysozyme crystallization is observed, for example, at around 3.5\% (w/v) $\mathrm{NaCl}$ in $20 \mathrm{mg} / \mathrm{ml}$ HEWL (denoted by a pair of plus and minus in Table 1). Figure 2 shows photographs of $30 \mathrm{mg} / \mathrm{ml}(2.1 \mathrm{mM})$ HEWL solutions in micro test tubes two days after the addition of 3.0\% (w/v) or 3.5\% (w/v) NaCl. No crystal growth is observed in the $3.0 \%(\mathrm{w} / \mathrm{v}) \mathrm{NaCl}$ solution, while in the $3.5 \%(\mathrm{w} / \mathrm{v}) \mathrm{NaCl}$ solution, many lysozyme crystals have grown $(N>100$ in $180 \mu \mathrm{l})$, which are tetragonal or orthorhombic crystals $<0.2 \mathrm{~mm}^{3}$ in volume.

Many experiments on protein crystallization indicate that a metastable state between precipitant and dissoluble regions is advantageous for obtaining good quality protein crystals [1] [2]. It is very important in protein crystallization to discern the difference between protein aggregations in the dissolving and the metastable areas from pre-crystalline solutions. The crystallization boundary for the measured lysozyme solutions (Table 1) nearly corresponds to a solubility curve in the phase diagram, for example, as presented in [4]. It is worth noting that the crystallizing lysozyme solutions around the boundary are not situated on the liquid-liquid phase-separation area. Information on the boundary of protein crystallization in the metastable region is useful for crystallization screening.

\subsection{Forward Static Light Scattering (F-SLS) Characteristics}

Figure 3 shows the log-log plots of $I_{\mathrm{s}}$ vs. $q$ from the SLS profiles at small angles for (a) $20 \mathrm{mg} / \mathrm{ml}$ HEWL solu-

Table 1. Crystallization conditions for lysozyme with $\mathrm{NaCl}$ at $20^{\circ} \mathrm{C}$.

\begin{tabular}{|c|c|c|c|c|c|c|c|c|c|c|}
\hline \multirow{2}{*}{$\begin{array}{l}\text { Concentration of Lysozyme } \\
\qquad(\mathrm{mg} / \mathrm{ml})\end{array}$} & \multicolumn{10}{|c|}{ Concentration of $\mathrm{NaCl}(\% \mathrm{w} / \mathrm{v})$} \\
\hline & 0 & 1.0 & 2.0 & 3.0 & 3.5 & 4.0 & 4.5 & 5.0 & 6.0 & 7.0 \\
\hline 30 & $-^{\mathrm{a}}$ & - & - & - & + & + & + & + & + & + \\
\hline 20 & - & - & - & - & +- & + & + & + & + & + \\
\hline 10 & - & - & - & - & - & - & +- & +- & + & + \\
\hline
\end{tabular}

a. Symbols: +, Crystallization; -, No Crystallization; +-, Crystallization or not.

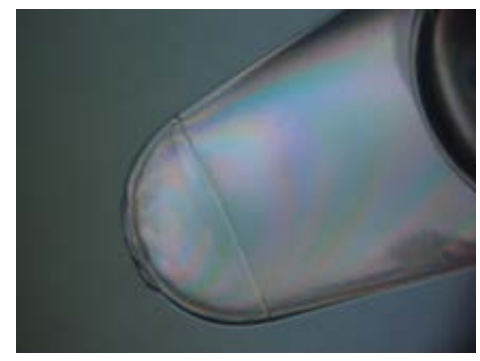

(a)

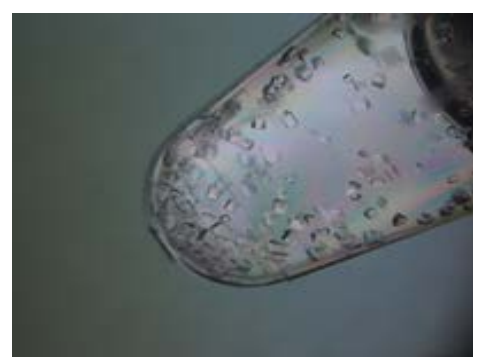

(b)

Figure 2. Photographs of the $30 \mathrm{mg} / \mathrm{ml}$ lysozyme solutions after 2 days with NaCl: (a) $3.0 \%(w / v)$ and (b) $3.5 \%(w / v)$. 


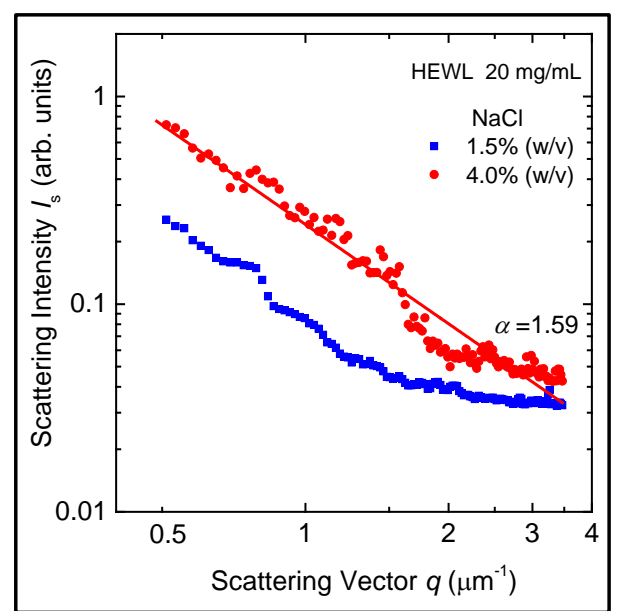

(a)

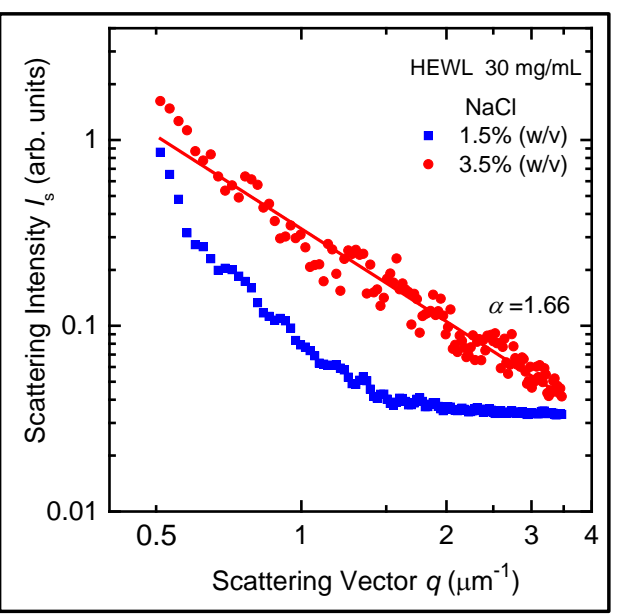

(b)

Figure 3. Log-log plots of scattering light intensity $I_{\mathrm{s}}$ as a function of scattering wave vector $q$ for (a) $20 \mathrm{mg} / \mathrm{ml}$ lysozyme solution with $1.5 \%(\mathrm{w} / \mathrm{v})$ and $4.0 \%(\mathrm{w} / \mathrm{v}) \mathrm{NaCl}$ and for (b) $30 \mathrm{mg} / \mathrm{ml}$ lysozyme solution with $1.5 \%(\mathrm{w} / \mathrm{v})$ and $3.5 \%(\mathrm{w} / \mathrm{v}) \mathrm{NaCl}$.

tions with $1.5 \%$ and $4.0 \%(\mathrm{w} / \mathrm{v}) \mathrm{NaCl}$ and for (b) $30 \mathrm{mg} / \mathrm{ml} \mathrm{HEWL}$ solutions with $1.5 \%$ and $3.5 \%(\mathrm{w} / \mathrm{v}) \mathrm{NaCl}$. The solution samples evaluated by F-SLS were in a state where microcrystal growth of lysozyme was unobservable using a microscope just after the F-SLS measurements. The addition of higher NaCl to the solutions at the same HEWL concentration exhibits a considerable increase in the F-SLS intensity. The enhanced F-SLS curve indicates formation of larger aggregates (clusters) of lysozyme, caused by the additional NaCl. The fluctuations in the SLS are not a noise signal, which are attributed to dynamics of lysozyme aggregates. The SLS log-log profiles of the higher $\mathrm{NaCl}$ addition can be fitted by a linear function with a negative slope giving a power law of $I_{s}(q) \propto q^{-\alpha}$. The power value $\alpha$ is $1.59 \pm 0.06$ for the $20 \mathrm{mg} / \mathrm{ml}$ solution with $4.0 \%(\mathrm{w} / \mathrm{v}) \mathrm{NaCl}$. The determination coefficient $R^{2}$ is 0.950 , as obtained from least-square fitting. For the $30 \mathrm{mg} / \mathrm{ml}$ solution with $3.5 \%(\mathrm{w} / \mathrm{v}) \mathrm{NaCl}, \alpha=1.66 \pm 0.05$ and $R^{2}$ is 0.955 . The $R^{2}$ gives a measure of how well the data are fitted by a curve $\left(0 \leq R^{2} \leq 1\right)$.

The power-law SLS pattern indicates a fractal aggregation of lysozyme in the pre-crystalline solutions. As this is well fitted by a linear function, the power value $\alpha$ gives a fractal dimension $D$ for the lysozyme fractal clusters, which gives information on the interior fractal structure [16]. In contrast with the higher $\mathrm{NaCl}$ solutions, the 1.5\% (w/v) $\mathrm{NaCl}$ solutions exhibit no power law in the SLS curve. This indicates that the lysozyme aggregates form in the $1.5 \%(\mathrm{w} / \mathrm{v}) \mathrm{NaCl}$ solutions are not fractal clusters. No crystals of lysozyme were grown in the $1.5 \%(\mathrm{w} / \mathrm{v})$ $\mathrm{NaCl}$ solutions after several weeks, while in the higher $\mathrm{NaCl}$ solutions the growth of lysozyme crystals were confirmed after two days, as shown in Table 1.

\subsection{Relationship between Aggregation in Pre-Crystalline Solutions and the Crystallization Conditions}

Although we could not interpret the power law for the SLS patterns of the $1.5 \%(\mathrm{w} / \mathrm{v}) \mathrm{NaCl}$ solutions in Figure 3 , the accuracy of the linear fit of the SLS data can act as a defacto metric. To this end, we estimated the power value $\alpha$ for the measured SLS data of the pre-crystalline solutions and evaluate the fit with $R^{2}$ values. Typical results are shown in Figure 4. At NaCl concentrations lower than $3 \%(\mathrm{w} / \mathrm{v})$ in the 20 and $30 \mathrm{mg} / \mathrm{ml} \mathrm{HEWL}$ solutions, the SLS curves could not be fitted to a linear function $\left(R^{2}<0.9\right.$, the broken line in Figure 4(b)). Thus we can confirm the formed aggregates are not fractal clusters in these conditions. Through this region, the $\alpha$ value increases and $R^{2}$ rises as the $\mathrm{NaCl}$ concentration increases. This behavior indicates that the added $\mathrm{NaCl}$ leads to lysozyme aggregation and the formation of fractal clusters.

In contrast, in the higher $\mathrm{NaCl}$ concentration region of $>4 \%(\mathrm{w} / \mathrm{v})$, the SLS patterns can be fitted to a power law $\left(R^{2}>0.9\right.$, as shown in Figure 4(b)), which means the aggregates are fractal clusters. As is shown in Table 1, the crystallization boundary exists between $3 \%$ and $4 \%(\mathrm{w} / \mathrm{v}) \mathrm{NaCl}$ in 20 and $30 \mathrm{mg} / \mathrm{ml} \mathrm{HEWL}$ solutions. Comparing the data in Figure 4 with the crystallization conditions, reveals that on the higher $\mathrm{NaCl}$ concentration side 


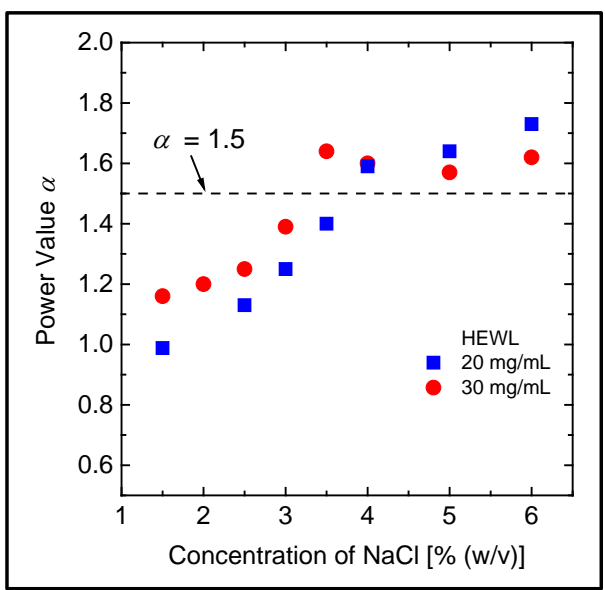

(a)

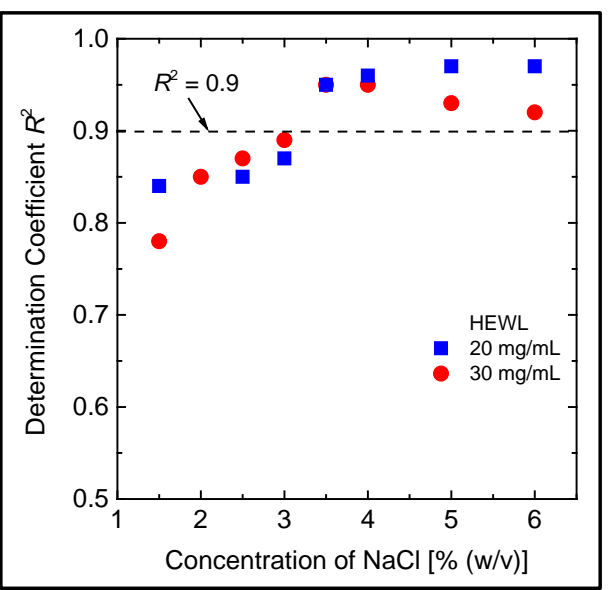

(b)

Figure 4. (a) Dependence of the power value $\alpha$ on the $\mathrm{NaCl}$ concentration when added to 20 and $30 \mathrm{mg} / \mathrm{ml}$ HEWL solutions, as estimated from the SLS profiles using least-square fitting. (b) Change in determination coefficient $R^{2}$ with the added $\mathrm{NaCl}$ concentrations.

of the crystallization boundary, the fractal dimensions $D$ (in this case, equal to $\alpha$ ) of the lysozyme clusters are larger than those of the lower concentration area and exceed a value of 1.5 (the broken line in Figure 4(a)). The comparison proves that the pre-crystalline solutions where lysozyme crystals grow exhibit the formation of fractal clusters with fractal dimensions over 1.5.

The fractal dimension $D$ is strongly related to the mass density of the fractal aggregate structure and the fractal aggregates can be characterized by the power-law dependence of an average local density $\langle\rho(r)\rangle_{0}$ at distance $r$ from a given point [16] [17]: $\langle\rho(r)\rangle_{0} \propto r^{D-d}$, where $d$ is a spatial dimension. The structure factor $S(q)$ obtained from static light scattering measurements of $I_{s}(q)$ is directly connected to $\langle\rho(r)\rangle_{0}$, and is given by the Fourier transform of $\langle\rho(r)\rangle_{0}$ [16]. Since the average local density $\langle\rho(r)\rangle_{0}$ expresses a spatial correlation between two arbitrary scattering sites in the clusters, the larger the correlation in the distances between scattering elements, such as particles forming fractal structures, the larger the $D$, which means that the structure of fractal clusters is denser.

The $D$ of fractal clusters formed in the crystallizing lysozyme solutions is of the range of $1.5 \sim 1.8$, as seen in Figure 4(a). The estimated $D$ values are not significantly different from those reported by Georgalis et al. [13] [14] or Tanaka et al. [7] [15]. For typical fractal aggregates like colloidal silica, gold and polystyrene particles, two types of fractal models are as well known: diffusion-limited cluster aggregation (DLCA) with $D \sim 1.8$ and reaction-limited cluster aggregation (RLCA) with $D \sim 2.1$ [16]-[19]. The fractal dimensions of the lysozyme clusters formed in the pre-crystalline solutions progressing to crystallizations are closer to the value of DLCA than RLCA. The lysozyme clusters are well hydrated and contain a considerable amount of $\mathrm{H}_{2} \mathrm{O}$, unlike fractal clusters of colloidal metals, silica, and polystyrene particles. Water-soluble protein crystals like lysozyme are typically made up of over $60 \%$ water [1]. Therefore, the pre-crystalline protein clusters probably have a low mass density as compared with colloidal particles, meaning the $D$ of the lysozyme clusters will be smaller. $d / 2<$ $D<d$ describes the range of $D$ known to undergo colloidal aggregation, as determined from simulations and experiments [16]. In the formation of the aggregates $d=3$, meaning the fractal dimension must be in the range, $1.5<D<3$. This agrees with the boundary value of $D=1.5$ found here for lysozyme fractal aggregates that lead to the formation of crystalline nuclei. These results indicate that high density fractal clusters of lysozyme with $D>1.5$ are formed in solutions which will progress to crystallization.

\section{Conclusion}

We have characterized lysozyme aggregates in pre-crystalline solutions using a highly sensitive FLS technique. The SLS profiles have been analyzed using a least-square fitting method and the fitting determination coefficient $R^{2}$, and this has been related to the conditions in which the lysozyme crystallizes because of added $\mathrm{NaCl}$. When the SLS patterns for the aggregates deviate from the power law or show low power values, the lysozyme solutions do not progress to crystallization. Pre-crystalline lysozyme solutions which do progress to crystallization 
generate dense fractal clusters with fractal dimensions of $D>1.5$. The forward-light-scattering method is a very powerful technique for screening protein crystal growth conditions.

\section{Acknowledgements}

The author thanks Prof. H. Koibuchi (Ibaraki National College of Technology) for useful discussions on fractals. This research was supported by the Salt Science Research Foundation and partly by JSPS KAKENHI.

\section{References}

[1] McPherson, A. (1982) Preparation and Analysis of Protein Crystals. Wiley, New York.

[2] Chayen, N.E. and Saridakis, E. (2008) Protein Crystallization: From Purified Protein to Diffraction-Quality Crystal. Nature Methods, 5, 147-153. http://dx.doi.org/10.1038/nmeth.f.203

[3] Muschol, M. and Rosenberger, F. (1997) Liquid-Liquid Phase Separation in Supersaturated Lysozyme Solutions and Associated Precipitate Formation/Crystallization. The Journal of Chemical Physics, 107, 1953-1962. http://dx.doi.org/10.1063/1.474547

[4] Tanaka, S., Yamamoto, M., Ito, K., Hayakawa, R. and Ataka, M. (1997) Relation between the Phase Separation and the Crystallization in Protein Solutions. Physical Review E, 56, R67-R69. http://dx.doi.org/10.1103/PhysRevE.56.R67

[5] Tanaka, S., Ataka, M. and Ito, K. (2002) Pattern Formation and Coarsening during Metastable Phase Separation in Lysozyme Solutions. Physical Review E, 65, 51804-1-6. http://dx.doi.org/10.1103/PhysRevE.65.051804

[6] Price, W.S., Tsuchiya, F. and Arata, Y. (1999) Lysozyme Aggregation and Solution Properties Studied Using PGSE NMR Diffusion Measuerments. Journal of the American Chemical Society. 121, 11503-11512. http://dx.doi.org/10.1021/ja992265n

[7] Tanaka, S., Ito, K., Hayakawa, R. and Ataka, M. (1999) Size and Number Density of Precrystalline Aggregates in Lysozyme Crystallization Process. The Journal of Chemical Physics, 111, 10330-10337. http://dx.doi.org/10.1063/1.480381

[8] Skouri, M., Lorber, B., Giegé, R., Munch, J. -P. and Candau, J. S. (1995) Effect of Macromolecular Impurities on Lysozyme Solubility and Crystallizability: Dynamic Light Scattering, Phase Diagram, and Crystal Growth Studies. Journal of Crystal Growth, 152, 209-220. http://dx.doi.org/10.1016/0022-0248(95)00051-8

[9] Muschol, M. and Rosenberger, F. (1995) Interactions in Undersaturated and Supersaturated Lysozyme Solutions: Static and Dynamic Light Scattering Results. The Journal of Chemical Physics, 103, 10424-10432. http://dx.doi.org/10.1063/1.469891

[10] Sazaki, G., Ooshima, H., Kato, J. Harano, Y. and Hirokawa, N. (1993) Mechanism of Crystallization of Enzyme Protein Thermolysin. Journal of Crystal Growth, 130, 357-367. http://dx.doi.org/10.1016/0022-0248(93)90521-W

[11] Eberstein, W., Georgalis, Y. and Saenger, W. (1994) Molecular Interactions in Crystallizing Lysozyme Solutions Studied by Photon Correlation Spectroscopy. Journal of Crystal Growth, 143, 71-78. http://dx.doi.org/10.1016/0022-0248(94)90369-7

[12] Tanaka, S. and Ataka, M. (2002) Protein Crystallization Induced by Polyethylene Glycol: A Model Study Using Apoferritin. The Journal of Chemical Physics, 117, 3504-3510. http://dx.doi.org/10.1063/1.1477456

[13] Georgalis, Y., Zouni, A., Eberstein, W. and Saenger, W. (1993) Formation Dynamics of Protein Precrystallization Fractal Clusters. Journal of Crystal Growth, 126, 245-260. http://dx.doi.org/10.1016/0022-0248(93)90031-Q

[14] Georgalis, Y., Umbach, P., Saenger, W., Ihmels, B. and Soumpasis, D.M. (1999) Ordering of Fractal Clusters in Crystallizing Lysozyme Solutions. Journal of the American Chemical Society, 121, 1627-1635. http://dx.doi.org/10.1021/ja982407y

[15] Tanaka, S., Yamamoto, M., Kawashima, K., Ito, K., Hayakawa, R. and Ataka, M. (1996) Kinetic Study on the Early Stage of the Crystallization Process of Two Forms of Lysozyme Crystals by Photon Correlation Spectroscopy. Journal of Crystal Growth, 168, 44-49. http://dx.doi.org/10.1016/0022-0248(96)00361-2

[16] Witten, T.A. and Pincus, P.A. (2004) Structured Fluids: Polymers, Colloids, Surfactants. Oxford, New York.

[17] Schaefer, D.W., Martin, J. E., Wiltzius, P. and Cannell, D.S. (1984) Fractal Geometry of Colloidal Aggregates. Physical Review Letters, 52, 2371-2374. http://dx.doi.org/10.1103/PhysRevLett.52.2371

[18] Weitz, D. A., Huang, J. S., Lin, M. Y. and Sung, J. (1985) Limits of the Fractal Dimension for Irreversible Kinetic Aggregation of Gold Colloids. Physical Review Letters, 54, 1416-1419. http://dx.doi.org/10.1103/PhysRevLett.54.1416

[19] Lin, M. Y., Lindsay, H.M., Weitz, D.A., Ball, R.C., Klein, R. and Meakin, P. (1989) Universality in Colloid Aggregation. Nature, 339, 360-362. http://dx.doi.org/10.1038/339360a0

[20] Wakamatsu, T. (2011) Forward Light Scattering for Highly Sensitive Detection of Aggregation in Crystallizing Protein 
Solutions. Applied Physics Letters, 98, 263701-1-3. http://dx.doi.org/10.1063/1.3603932

[21] Wakamatsu,T., Toyoshima, S. and Shimizu, H. (2011) Observation of Electric-Field Induced Aggregation in Crystallizing Protein Solutions by Forward Light Scattering. Applied Physics Letters, 99, 153701-1-3.

http://dx.doi.org/10.1063/1.3648114. 